\title{
DESIGN OF IIR EIGENFILTERS WITH ARBITRARY MAGNITUDE FREQUENCY RESPONSE
}

\author{
F. Argenti and E. Del Re \\ Dipartimento di Ingegneria Elettronica, Università di Firenze \\ Via di Santa Marta, 3 - 50139 Firenze - Italy \\ Tel. +39554796 424, Fax +39554796 485 \\ e-mail: argenti@cosimo.die.unifi.it
}

\begin{abstract}
In this study, the eigenfilter approach is applied to designing Infinite Impulse Response (IIR) filters having an arbitrary magnitude frequency response. A causal rational transfer function having an arbitrary number of poles and zeros is achieved. The procedure works in the frequency domain. Some numerical examples showing the application of the presented method to the design of multiband filters with different gains and different magnitude shape in each band are presented.
\end{abstract}

\section{INTRODUCTION}

The eigenfilter approach is an appealing way of designing digital filters, mainly because of the simplicity of its implementation. In fact, it can be applied to designing several types of Finite Impulse Response (FIR) or IIR filters [1]-[8]. The method consists in expressing the error between a target and a digital filter response as a real, symmetric, positive-definite quadratic form in the filter coefficients. The error can be referred either to the time, the frequency domain, or both of them. The eigenvector corresponding to the minimum eigenvalue yields the optimum filter coefficients according to the chosen error measure. Applying the eigenfilter approach to the IIR case is more difficult than in the FIR case. Design of IIR eigenfilters in the time domain has been addressed in [5]. A disadvantage of such approach is that it is more difficult to define a frequency weighting function. In [6][7] the eigenfilter approach is applied to the design of allpass sections with a given phase response. By summing up suitable allpass functions a lowpass or multiband frequency response filter can be designed [7][8]. In this case, however, the degrees of the numerator and the denominator of the global transfer function are related to the degrees of the allpass sections composing the system and can not be completely arbitrary. In [9] the solution of an eigenvalue problem yields the IIR filter coefficients, even though the classical eigenfilter approach, based on the Rayleigh's principle [1] and on the search for the minimum eigenvalue of a positive-definite matrix, is not used.

In this study the eigenfilter approach is used to design causal IIR filters with an arbitrary number of zeros and poles, whose magnitude frequency response can be arbitrarily shaped. The method and the results that will be shown represent an improvement of the work presented in [10]. The examples that will be given in the experimental results section will illustrate how the proposed method works to design multiband filters with an arbitrary magnitude frequency response.

\section{EIGENFILTER APPROACH TO DESIGN IIR FILTERS}

The main problem in designing IIR eigenfilters is expressing a measure which indicates the difference between a target function and the filter frequency response as a quadratic form in the filter coefficients. This task is easier in the FIR case, where the transfer function is not rational.

Let $H(z)$ be a rational function having $M$ zeros and $N$ poles (with arbitrary $M$ and $N$ ), i.e.,

$$
H(z)=\frac{b_{0}+b_{1} z^{-1}+\ldots+b_{M} z^{-M}}{a_{0}+a_{1} z^{-1}+\ldots+a_{N} z^{-N}}=\frac{N(z)}{D(z)}
$$

where $a_{i}, i=0,1, \ldots, N$, and $b_{i}, i=0,1, \ldots, M$, are real coefficients. Let $H^{d}(\omega)$ be a target function representing, in the simplest cases, a lowpass, highpass, bandpass filter frequency response. Suppose $H^{d}(\omega)$ is defined in a generic interval $I_{k}=\left(\omega_{k}, \omega_{k+1}\right)$ as $H_{k}^{d}(\omega)=$ $f_{k}(\omega) e^{j \varphi_{k}(\omega)}$, where $f_{k}(\omega)$ is a given real function. Consider we are interested in approximating only the magnitude of the target function $H^{d}(\omega)$, so that $\varphi_{k}(\omega)$ can be any arbitrary real function. In the absence of other information we could assume $\varphi_{k}(\omega)=K \omega$, with $K$ a 
given constant. In general, $H^{d}(\omega)$ is approximated by $H(\omega)=\left.H(z)\right|_{z=e^{j \omega}}$ so that the error function

$$
\varepsilon(\omega)=H^{d}(\omega)-H(\omega)
$$

is minimized in some sense. The function $\varepsilon(\omega)$ is not linear in the $a_{i}$ 's and $b_{i}$ 's and therefore $\|\varepsilon(\omega)\|_{2}$ is not a quadratic form in the filter coefficients as in the FIR case. Therefore, a new function related to $\varepsilon(\omega)$ must be used. Consider

$$
E(\omega)=\varepsilon(\omega) D(\omega)
$$

and let $E_{k}(\omega)$ be the function $E(\omega)$ in the interval $I_{k}$, that is

$$
\begin{aligned}
E_{k}(\omega)= & H_{k}^{d}(\omega)\left(a_{0}+a_{1} e^{-j \omega}+\ldots+a_{N} e^{-j N \omega}\right)- \\
& -b_{0}-b_{1} e^{-j \omega}-\ldots-b_{M} e^{-j M \omega}
\end{aligned}
$$

The function $D(\omega)$ depends on the poles of $H(z)$. For $\omega$ in the passband, $e^{j \omega}$ is close to the poles of $H(z)$ and $|D(\omega)|$ can take on small values. However, since poles can not be placed on the unit circle, $|D(\omega)|$ is always nonzero valued in useful filters. Minimizing $E(\omega)$ in some sense means a weighted minimization of the error function $\varepsilon(\omega)$, where $D(\omega)$ acts as the weighting function. Lower weight is assigned to regions in correspondence to the poles closer to the unit circle, but, since a nonzero weighting function is used, infinite or a very large value of the error $\varepsilon(\omega)$ at these frequencies is not expected. Consider the global cost function $\Phi$ given by

$$
\Phi=\sum_{k} \beta_{k} \phi_{k}
$$

where $\beta_{k}$ is a positive constant that weights the $k$-th interval cost function $\phi_{k}$ given by

$$
\begin{aligned}
\phi_{k}= & \int_{-\omega_{k+1}}^{-\omega_{k}}\left|E_{k}(\omega)\right|^{2} W_{k}(\omega) d \omega+ \\
& +\int_{\omega_{k}}^{\omega_{k+1}}\left|E_{k}(\omega)\right|^{2} W_{k}(\omega) d \omega
\end{aligned}
$$

where $W_{k}(\omega)$ is a positive weighting function. If we define

$$
\begin{aligned}
& \mathbf{C}_{k}(\omega)=\left[\begin{array}{ll}
H_{k}^{d}(\omega) \mathrm{e}_{\mathbf{N}}(\omega) & \left.-\mathrm{e}_{\mathbf{M}}(\omega)\right]^{T}
\end{array}\right. \\
& \mathbf{A}=\left[\begin{array}{llllllll}
a_{0} & a_{1} & \ldots & a_{N} & b_{0} & b_{1} & \ldots & b_{M}
\end{array}\right]^{T}
\end{aligned}
$$

where $\mathbf{e}_{\mathbf{L}}(\omega)=\left[1 e^{-j \omega} \ldots e^{-j L \omega}\right]^{T}$, then the cost function $\phi_{k}$ can be expressed as

$$
\begin{aligned}
\phi_{k}= & \int_{-\omega_{k+1}}^{-\omega_{k}} \mathbf{A}^{T} \mathbf{C}_{k}^{*}(\omega) \mathbf{C}_{k}^{T}(\omega) \mathbf{A} W_{k}(\omega) d \omega+ \\
& +\int_{\omega_{k}}^{\omega_{k+1}} \mathbf{A}^{T} \mathbf{C}_{k}^{*}(\omega) \mathbf{C}_{k}^{T}(\omega) \mathbf{A} W_{k}(\omega) d \omega
\end{aligned}
$$

where superscript $\mathrm{T}$ and ${ }^{*}$ denote the transposition and conjugation operations, respectively $\left(\mathbf{A}^{*}=\mathbf{A}\right.$ since the filter coefficients are assumed real). Therefore, $\phi_{k}$ is given by

$$
\phi_{k}=\mathbf{A}^{T} \mathbf{P}_{k} \mathbf{A}
$$

where

$$
\begin{aligned}
\mathbf{P}_{k}= & \int_{-\omega_{k+1}}^{-\omega_{k}} \mathbf{C}_{k}^{*}(\omega) \mathbf{C}_{k}^{T}(\omega) W_{k}(\omega) d \omega+ \\
& +\int_{\omega_{k}}^{\omega_{k+1}} \mathbf{C}_{k}^{*}(\omega) \mathbf{C}_{k}^{T}(\omega) W_{k}(\omega) d \omega
\end{aligned}
$$

is a symmetric, real, positive-definite $(M+N+2) \times$ $(M+N+2)$ matrix. The global cost function $\Phi$ can be expressed as

$$
\Phi=\mathbf{A}^{T}\left(\sum_{k} \beta_{k} \mathbf{P}_{k}\right) \mathbf{A}=\mathbf{A}^{T} \mathbf{P A} .
$$

By using the eigenfilter approach, the optimal filter coefficients that minimize the cost function $\Phi$ are the elements of the eigenvector of the matrix $P$ corresponding to the minimum eigenvalue. The computation of the matrices $\mathbf{P}_{k}$ can be performed numerically in each band $\left(\omega_{k}, \omega_{k+1}\right)$.

The procedure that has been described gives the coefficients that minimize the cost function in a weighted least-square sense. However, the designed frequency response may have a behavior not completely satisfactory. The reasons why this happens and suitable countermeasures are now discussed.

In this study we are interested in approximating only the magnitude of $H^{d}(\omega)$. However, the cost function $E(\omega)$ also depends on $\varphi(\omega)$, i.e., the phase of $H^{d}(\omega)$. Therefore, different frequency responses can be obtained according to the choice of $\varphi(\omega)$. In the absence of any information we could assume $\varphi(\omega)=K \omega$, where $K$ is a given constant. This choice, hovever, leads to an amplitude frequency response that can considerably differ from the target function $\left|H^{d}(\omega)\right|$. An iterative procedure we have found to be effective to reach well-behaved filter frequency responses is the following. Let $\mathbf{A}^{(n)}$ be the coefficient vector at the $\mathrm{n}$-th step and let $H^{(n)}(\omega)$ be the corresponding frequency response. Let $\varphi^{(n)}(\omega)$ be the phase of $H^{d}(\omega)$ at the n-th step. Suppose $\varphi^{(n)}(\omega)=\angle H^{(n-1)}(\omega)$ and compute the coefficient vector $\mathbf{A}^{(n)}$ by solving the eigenfilter problem. At the first step $\varphi^{(0)}(\omega)$ is assumed linear.

A further improvement can be obtained by including within the procedure a function of the error computed at the $n$-th step. In previous articles [1][4][7] a recursive updating of the weighting function was introduced to obtain an almost equiripple frequency response. This approach can be used here to reduce amplitude errors. If we define the magnitude error in the 
interval $I_{k}$ at the n-th iteration as

$$
e_{k}^{(n)}(\omega)=|| H_{k}^{d}(\omega)|-| H_{k}^{(n)}(\omega)|| .
$$

then the weighting function to be used in the $(n+1)$-th iteration is

$$
W_{k}^{(n+1)}(\omega)=W_{k}^{(n)}(\omega) \operatorname{env}\left(e_{k}^{(n)}(\omega)\right)
$$

where env $(g(x))$ is the envelope of the positive function $g(x)$ (we have used a linear interpolation between the local maxima of $g(x)$ ). By using this approach, larger weights are assigned to frequencies where larger magnitude errors occur. The function $W_{k}^{(n)}(\omega)$ is normalized at each step so that $1 /(2 \pi) \int_{I_{k}} W_{k}^{(n)}(\omega) d \omega=1$. The choice of the $\beta_{k}$ 's determines the weights to be assigned to the different intervals.

\section{EXPERIMENTAL RESULTS}

The effectiveness of the presented method can be shown through some numerical examples. The integrals that appear into the definition of the matrices $\mathbf{P}_{k}$ have been computed numerically by using a grid of 100 points in each band. The iterative procedure stops when $\max (\mid$ $\left.\mathbf{A}^{(n+1)}-\mathbf{A}^{(n)} \|\right)<\epsilon_{1}$ or $\max _{k} \max _{\omega}\left(|| H_{k}^{(n+1)}(\omega) \mid-\right.$ $\left.\left|H_{k}^{(n)}(\omega)\right| \mid\right)<\epsilon_{2}$ (in our design examples we used $\epsilon_{1}=$ $10^{-4}$ and $\epsilon_{1}=10^{-5}$ ). The filter characteristics are given in the frequency domain by using the normalized frequency $F=\omega /(2 \pi)$.

Example 1. Lowpass filter with passband for $0 \leq 1$ $F \mid \leq 0.1$ and stopband for $0.12 \leq|F| \leq 0.5$. The transition band is considered as a "don't care" band. By using $M=8$ and $N=5$ we have obtained the result shown in Fig. 1. The weights $\left\{\beta_{k}\right\}=\{1,1\}$ were used.

Example 2. Multiband filter with a linearly shaped passband for $0 \leq|F| \leq 0.2$, a constant gain (equal to $0.7)$ passband for $0.4 \leq|F| \leq 0.5$ and an attenuation band for $0.25 \leq|F| \leq 0.35$. The transition band are shaped with a fifth order polynomial with null derivatives at the points where the adjacent passband and stopband are joined. The target function is shown in Fig. 2 with solid line. By using $M=11$ and $N=7$ we have obtained the frequency response shown in Fig. 2 with dashed line. The weights $\left\{\beta_{k}\right\}=\{1,1,5,1,5\}$ were used (the weights refer to passbands, transition bands and attenuation band in the order of increasing frequencies). Fig. 3 reports the designed magnitude frequency response in logarithmic scale.

Example 3. Multiband filter with a constant gain (equal to 1) passband for $0 \leq|F| \leq 0.1$, a constant gain (equal to 0.5 ) passband for $0.13 \leq|F| \leq 0.3$ and an attenuation band for $0.33 \leq|F| \leq 0.5$. Also in this case fifth order polynomial shaped transition bands have been used. The target function and the frequency response obtained with $M=15$ and $N=9$ are shown in Fig. 4 with solid line and dashed line, respectively. The designed magnitude frequency response in logarithmic scale is shown in Fig. 5. The weights $\left\{\beta_{k}\right\}=\{5$, $1,5,1,10\}$ were used.

As can be seen, in all the cases that are presented the target function has been closely approximated by the designed magnitude frequency responses.

\section{CONCLUSIONS}

In this study a method for applying the eigenfilter approach, based on the Rayleigh's principle, to the design of IIR digital filters is shown. The method works in the frequency domain and allows to design filters with an arbitrary magnitude frequency response. The number of zeros and poles of the filter transfer function is also arbitrary.

\section{REFERENCES}

[1] P.P. Vaidyanathan, T.Q. Nguyen, "Eigenfilters: A New Approach to Least-Squares FIR Filter Design and Applications Including Nyquist Filters", IEEE Trans. Circuits Syst., Vol. CAS-34, no. 1, Jan. 1987, pp. 11-23.

[2] S.C. Pei, J.J. Shyu, "Design of FIR Hilbert Transformers and Differentiators by Eigenfilter", IEEE Trans. Circuits Syst, Vol. 35, no. 11, Nov. 1988, pp. 1457-1461.

[3] S.C. Pei, J.J. Shyu, "Eigenfilter Design of HigherOrder Digital Differentiators", IEEE Trans. Signal Processing, Vol. 37, no. 4, Apr. 1989, pp. 505-511.

[4] T.Q. Nguyen, "The Design of Arbitrary FIR Digital Filters Using the Eigenfilter Method", IEEE Trans. Signal Processing, Vol. 41, no. 3, Nov. 1993, pp. 1128-1139.

[5] S.C. Pei, J.J. Shyu, "Design of 1-D and 2-D IIR Eigenfilters", IEEE Trans. Signal Processing, Vol. 42, no. 4, Apr. 1994, pp. 962-966.

[6] S.C. Pei, J.J. Shyu, "Eigenfilter Design of 1-D and 2-D IIR Digital All-Pass Filters", IEEE Trans. Signal Processing, Vol. 42, no. 4, Apr. 1994, pp. 966968.

[7] T.Q. Nguyen, T.I. Laakso, R.D. Koilpillai, "Eigenfilter Approach for the Design of Allpass Filters Approximating a Given Phase Response", IEEE 
Trans. Signal Processing, Vol. 42, no. 9, Sep. 1994, pp. 2257-2263.

[8] S.C. Pei, J.J. Shyu, "Design of IIR multiband filters using IIR all-pass filters", Signal Processing, Vol. 37, 1994, pp. 87-94.

[9] X. Zhang, H. Iwakura "Desing of IIR Digital Filters Based on Eigenvalue Problem", IEEE Trans. Signal Processing, Vol. 44, no. 6, June 1996, pp. 1325-1333.

[10] F. Argenti, E. del Re, "Design of IIR eigenfilters in the frequency domain", 13th International Conference on Digital Signal Processing DSP'97, Santorini, Greece, 2-4 July, 1997, pp. 629-632.

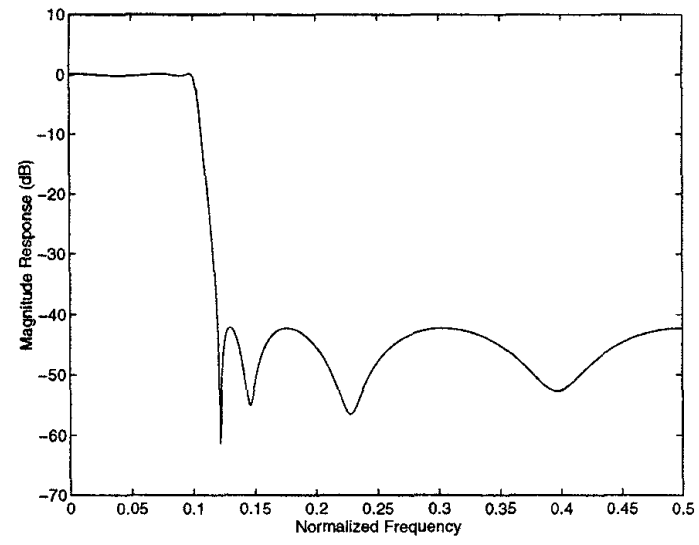

Figure 1: Example 1. Frequency response of the filter designed with orders $M=8$ and $N=5$.

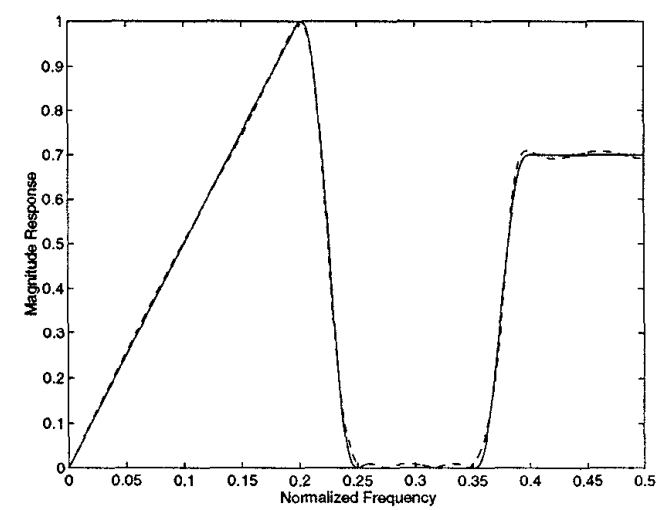

Figure 2: Example 2. Target function (solid line) and designed frequency response obtained with orders $M=$ 11 and $N=7$ (dashed line).

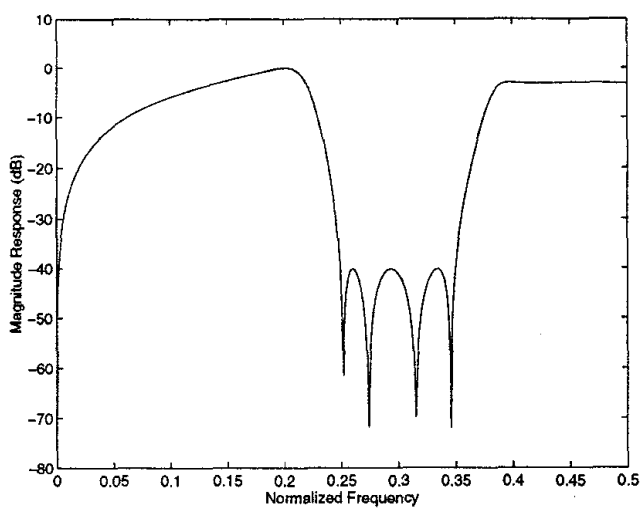

Figure 3: Example 2. Designed frequency response in logarithmic scale.

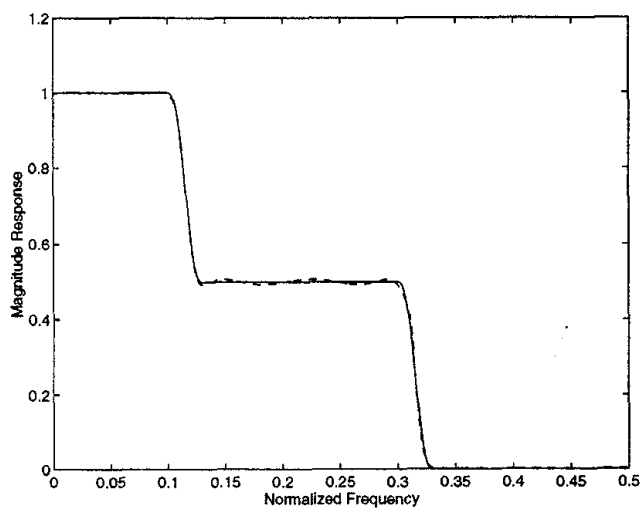

Figure 4: Example 3. Target function (solid line) and designed frequency response obtained with orders $M=$ 15 and $N=9$ (dashed line).

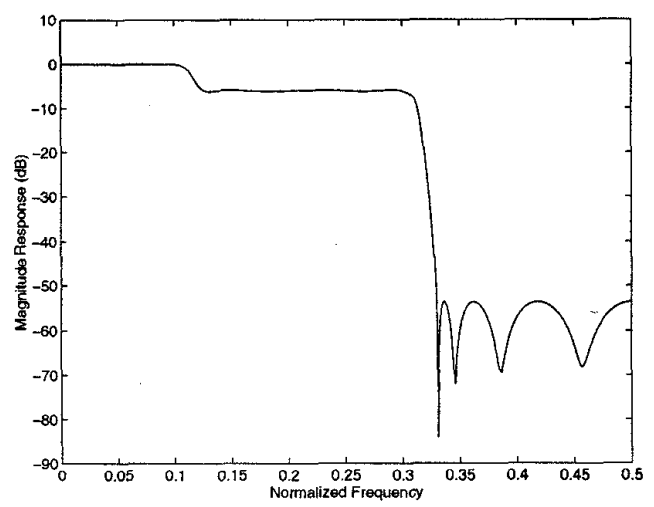

Figure 5: Example 3. Designed frequency response in logarithmic scale. 